\title{
Unusual Location of a Cardiac Papillary Fibroelastoma
}

\author{
George Samanidis ${ }^{\mathrm{a}, \mathrm{c}}$, Charalampos Katselis ${ }^{\mathrm{a}}$, Constantinos Contrafouris ${ }^{\mathrm{a}}$, \\ Theofani Antoniou ${ }^{\mathrm{b}}$, Konstantinos Perreas ${ }^{\mathrm{a}}$
}

\begin{abstract}
Cardiac papillary fibroelastoma is a rare benign cardiac tumor that represents less than $10 \%$ of all cardiac tumors. Most papillary fibroelastomas are relatively small. Symptoms, if present, are due to flow obstruction or peripheral embolization. The most common sites of origin are aortic and mitral valve surfaces. We present successful surgical resection of a papillary fibroelastoma through the mitral valve which originated from the posterior papillary muscle of the left ventricle.
\end{abstract}

Keywords: Cardiac tumor; Cardiac fibroelastoma; Cardiac surgery

\section{Introduction}

Cardiac papillary fibroelastoma (CPF) is a rare benign cardiac tumor and location in the left ventricle (LV) is noted in $9 \%$ of cases [1]. The symptoms, if present, depend on location and size. When diagnosis is established, surgical resection is indicated to establish diagnosis, to prevent complications such peripheral embolization, heart failure and syncoptic episodes.

\section{Case Report}

A 73-year-old male referred to our department for the evaluation of a suspected LV mass diagnosed by checkup transthoracic echocardiography (TTE) (Fig. 1a, b). The patient was asymptomatic. The TTE futures showed a pedunculated mass localized in the LV and originating from the posterior papillary muscle. Intra-operative transesophageal echocardiography (TEE) confirmed the findings from TTE (Fig. 1c, d). The patient underwent surgical excision of the tumor with standard median sternotomy and cardiopulmonary bypass (CPB).

Manuscript submitted October 24, 2017, accepted November 10, 2017

aFirst Department of Adult Cardiac Surgery, Onassis Cardiac Surgery Center, Athens, Greece

bepartment of Anesthesiology, Onassis Cardiac Surgery Center, Athens, Greece

${ }^{\mathrm{c} C}$ Corresponding Author: George Samanidis, Onassis Cardiac Surgery Center, 356 Syggrou Av., 17674, Athens, Greece. Email: gsamanidis@yahoo.gr

doi: https://doi.org/10.14740/cr624w
Ascending aorta and bicaval cannulation was performed and following cardioplegic myocardial arrest approach of the posterior papillary muscle and the tumor was achieved through left atrium (LA) atriotomy and across the mitral valve. Complete resection of the mass with peduncle was performed (Fig. $2 \mathrm{a}, \mathrm{b})$. The patient was weaned from bypass without complications. After uneventful postoperative period, the patient was discharged at home on postoperative day 6. Pathological examination revealed that the mass was a CPF (Fig. 2c, d). At 3 months years follow-up, she is asymptomatic without neurological residue and the echo showed absence of recurrence.

\section{Discussion}

$\mathrm{CPF}$ is a rare cardiac tumor and it is the third common primary benign tumor after myxoma and lipoma [2]. Then most frequent site of origin of the $\mathrm{CPF}$ is the heart valve and location in the papillary muscle is extremely rare. CPF must therefore be included in the differential diagnosis of heart valve disease, metastatic neoplasm, bacterial endocarditis and endocardiac thrombus.

In the majority of cases, patients with CPF have asymptomatic course. If indeed symptoms are present, these result from the size and mobility of the CPF and its relation to surrounding structures. The most common mechanism of the symptoms is central or peripheral embolization from the tumor or the thrombus which forms on the tumor. Left-sided located CPFs cause neurological complications and are seen in 53\% of patients. In addition, acute coronary syndrome, mesenteric ischemia, renal infraction and sudden death have been reported [3]. Right-side location can cause pulmonary embolism.

Nowadays two- and three-dimensional echocardiography is a safe and probably more accurate method of diagnosing cardiac tumors and CPF is no exception [4]. In some instances, visualization can be enhanced by nuclear magnetic resonance imaging (MRI) and it should be considered when TEE is inconclusive. Coronary angiography or CT coronary angiography in patients over 40 years of age is generally required to rule out concomitant coronary artery disease.

When a clinical diagnosis of CPF has been made, surgical resection should be performed for preventing complications from central and peripheral embolization. Surgical excision for treating CPF is gold standard technique and approach of the tumor depends from location in the heart. CPB should be used in all cases and simple excision of the tumor with peduncle is enough in most patients. However, when CPF located in heart valves, replacement or repair should be performed. Surgical 


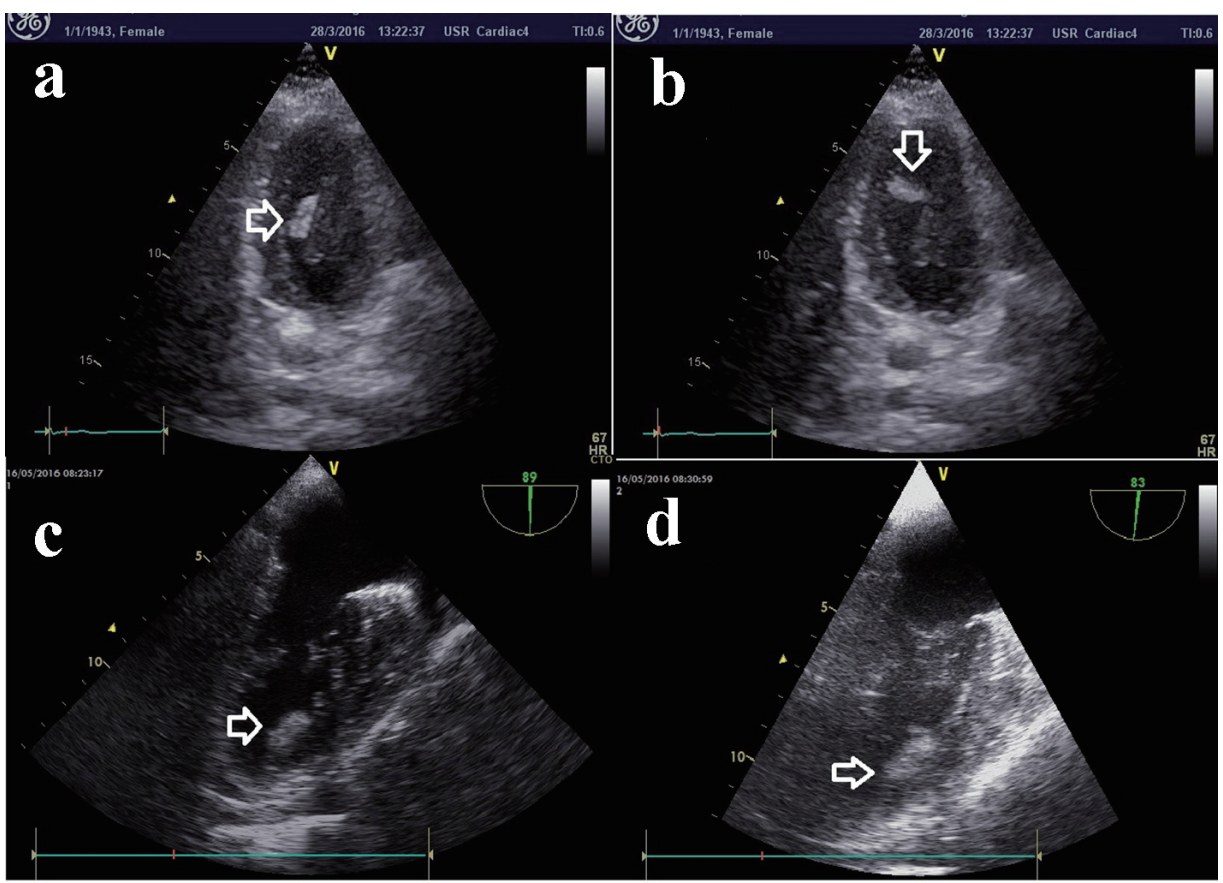

Figure 1. Pedunculated mass localized in the left ventricle and originating from the posterior papillary muscle (white arrow) (a, b) Preoperative transthoracic echocardiography findings. (c, d) Intra-operative transesophageal echocardiography findings.

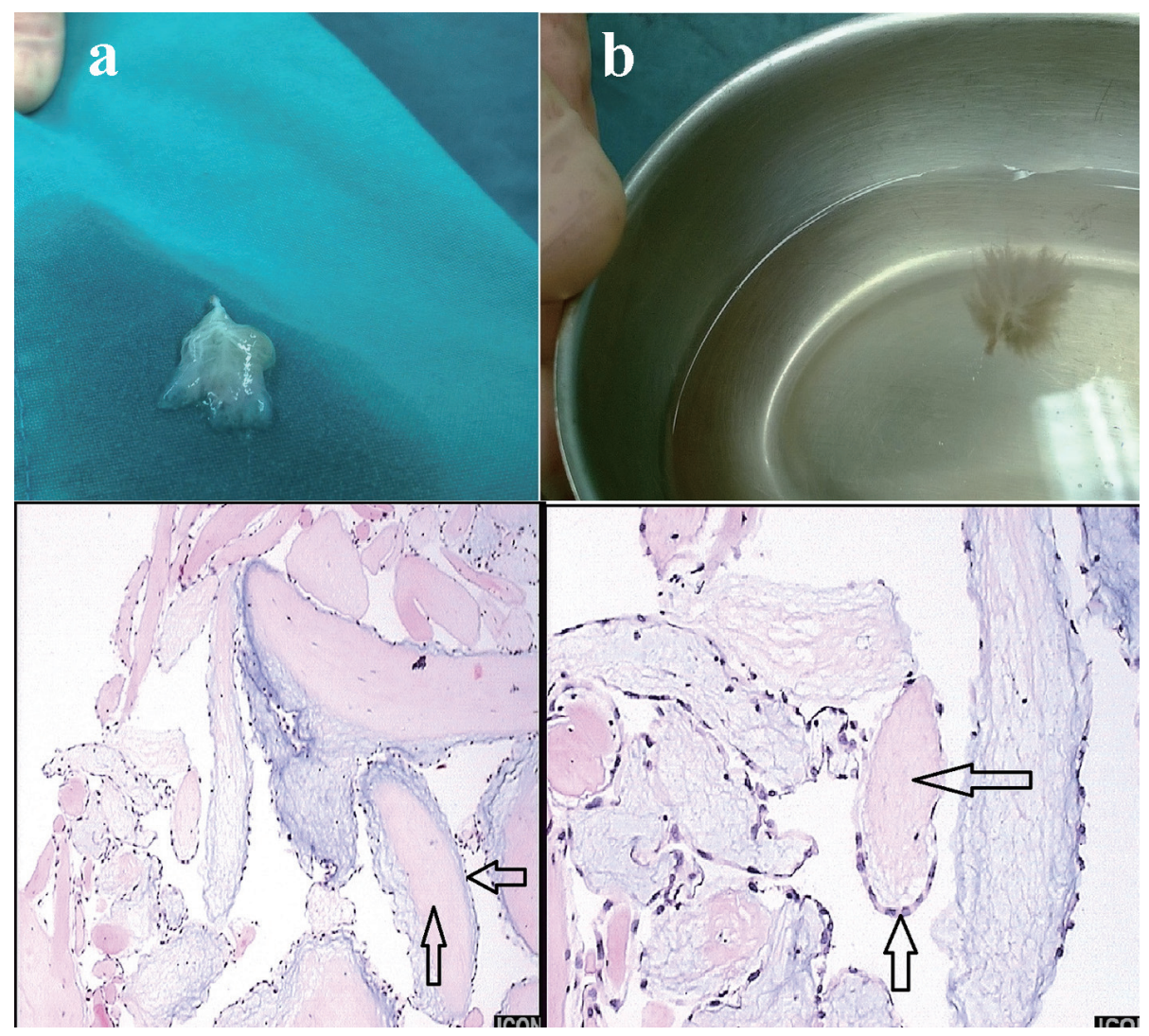

Figure 2. Complete resection of the mass with peduncle was performed. (a, b) Macroscopic appearance. (c, d) Microscopic appearance (pedunculated avascular collagen tumor (black arrow) covered by single layer of endothelium (black arrow)). 
excision of CPF has a low operative risk and gives excellent short- and long-term results. Recurrent CPF after surgical excision is uncommon.

\section{Conflict of Interest}

None.

\section{Reference}

1. Gowda RM, Khan IA, Nair CK, Mehta NJ, Vasavada BC, Sacchi TJ. Cardiac papillary fibroelastoma: a comprehensive analysis of 725 cases. Am Heart J. 2003;146(3):404-
410.

2. Howard RA, Aldea GS, Shapira OM, Kasznica JM, Davidoff R. Papillary fibroelastoma: increasing recognition of a surgical disease. Ann Thorac Surg. 1999;68(5):18811885.

3. Ngaage DL, Mullany CJ, Daly RC, Dearani JA, Edwards WD, Tazelaar HD, McGregor CG, et al. Surgical treatment of cardiac papillary fibroelastoma: a single center experience with eighty-eight patients. Ann Thorac Surg. 2005;80(5):1712-1718.

4. de Virgilio C, Dubrow TJ, Robertson JM, Siegel S, Ginzton L, Nussmeier M, Nelson RJ. Detection of multiple cardiac papillary fibroelastomas using transesophageal echocardiography. Ann Thorac Surg. 1989;48(1):119121. 